\title{
Optical Fiber Sensor of Partial Discharges in High Voltage DC Experiments
}

\author{
I. Búa-Núñez*a, C. G. Azcárraga-Ramos ${ }^{\mathrm{b}}$, J. E. Posada-Román ${ }^{\mathrm{a}}$ and J. A. Garcia-Souto*a \\ ${ }^{a}$ Electronics Technology Department, GOTL, Univ. Carlos III de Madrid, Av. Universidad 30, \\ 28911 Leganés, Spain; ${ }^{b}$ Department of Electrical Engineering, Università di Bologna, Bologna, Italy.
}

\begin{abstract}
A setup simulating High Voltage DC (HVDC) transformers barriers was developed to demonstrate the effectiveness of an optical fiber (OF) sensor in detecting partial discharges (PD) under these peculiar conditions. Different PD detection techniques were compared: electrical methods, and acoustic methods. Standard piezoelectric sensors (R15i-AST) and the above mentioned OF sensors were used for acoustic detection. The OF sensor was able to detect PD acoustically with a sensitivity better than the other detection methods. The multichannel instrumentation system was tested in real HVDC conditions with the aim of analyzing the behavior of the insulation (mineral oil/pressboard).
\end{abstract}

Keywords: Optical Fiber Sensor, Acoustic Detection, Partial Discharge, High Voltage DC (HVDC), LabVIEW.

\section{INTRODUCTION}

In current society, the supply of electrical energy is a concept of vital importance, being necessary to guarantee it at any time and place. Therefore, it is essential to apply maintenance strategies involving the monitoring, analysis and diagnosis of plant and equipment for the generation, transmission and distribution of energy.

The interest in the measurement of PD has been increased in recent years because PD represent a very reliable indicator of the degradation of electrical insulation. For this reason, the monitoring of that activity is very interesting with the aim of analyzing high-voltage electrical equipment such as transformers and studying the probability of failure.

Traditionally when talking about high voltage transformers it is assumed that they are AC transformers. For this reason, the most of studies are focused on AC voltage. However, use of HVDC technology for transmission in power grids is becoming more common [1], so that HVDC transformers are a key part. The reliability of the link depends on the insulation of the HVDC transformer [2]. In this paper an acoustic analysis of PD and the use of OF sensors are applied to HVDC system.

Within the methods of PD detection in transformers (electrical, RF, acoustic, chemical, optical, etc.), acoustic detection has major advantages regarding electromagnetic interference immunity, provide information for locating the acoustic emission source and it is easy to install in field [3-5].

Currently acoustic techniques using OF sensors are being developed [6-9]. There are some studies in this field but not many demonstrations with real PD signals. These sensors are embedded in the insulating medium, which can detect acoustic signal without the influence of the walls which causes undesirable effects like signal distortion and strong attenuation (90\%). Placing these sensors in the right place, they can provide information on the type and location of PD.

\section{SETUP FOR THE HVDC GENERATION AND THE OF DETECTION OF PD}

The objective of the study of PD in HVDC conditions is to analyze the behavior of the isolates (mineral oil/pressboard) to these stimuli. To do this, a test platform was constructed. The device under test (DUT) has a structure planepressboard-plane as shown in Fig. 1a). This structure goes immersed in a cubic tank of mineral oil. Fig. 1b) is a detail of the test setup of PD.

Due to the conditions of this experiment, the generated PD signals are very weak; hence it is very important to include highly sensitive sensors that, in addition, can be located close to the emission source. OF sensors fulfill the requirements, since they can be embedded in the insulating medium closer to the source, which allow detecting a stronger acoustic signal. The location of the OF sensor is illustrated in Figure $2 b$ ).

*\{ibua;jsouto\}@ing.uc3m.es; phone+34 91624 9907; portal.uc3m.es/portal/page/portal/dpto_tecnologia_electronica 


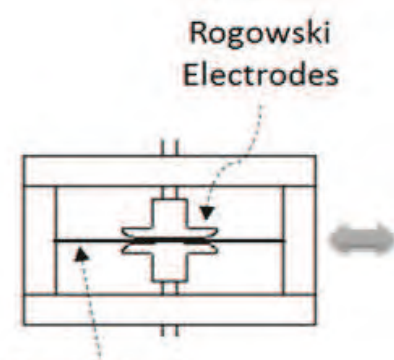

Pressboard

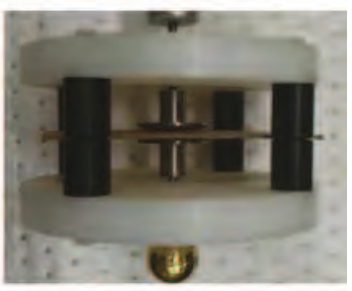

a)

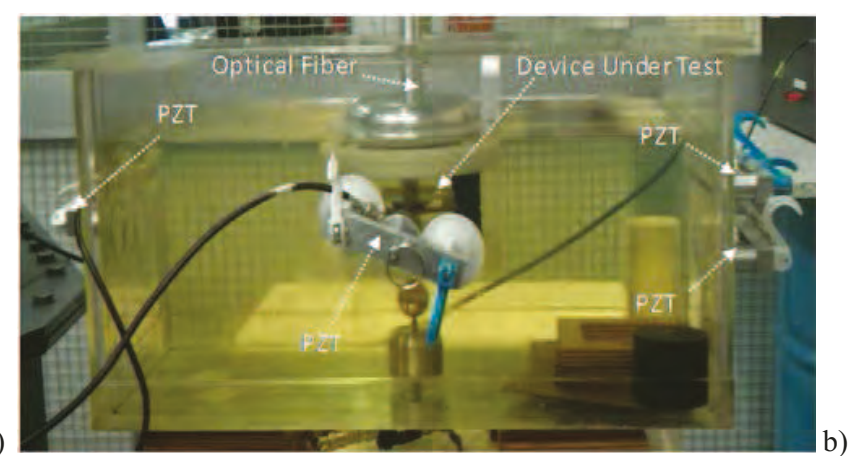

b)

Figure 1. a) Device under test (structure: plane-pressboard-plane). b) Test platform of PD.

The instrumentation scheme for the optical fiber sensor has been designed in a previous work [10] and it is shown in Figure 2a). This scheme consists of a sensor head of single-mode fiber at $633 \mathrm{~nm}$ that is sensitive to $150 \mathrm{kHz}$ acoustic emission. The optoelectronic condition system is illuminated by a He-Ne laser.

The conditioning system is based on an interferometer, an electro-mechanical actuator to compensate disturbances of low frequency, an amplifier of transimpedance with two detectors in differential configuration and a stage of band-pass filtering. This device provides a voltage output proportional to the optical phase which is in turn proportional to the acoustic pressure [10].

The characteristics of the OF sensing head used for this experiment are summarized in Table 1.
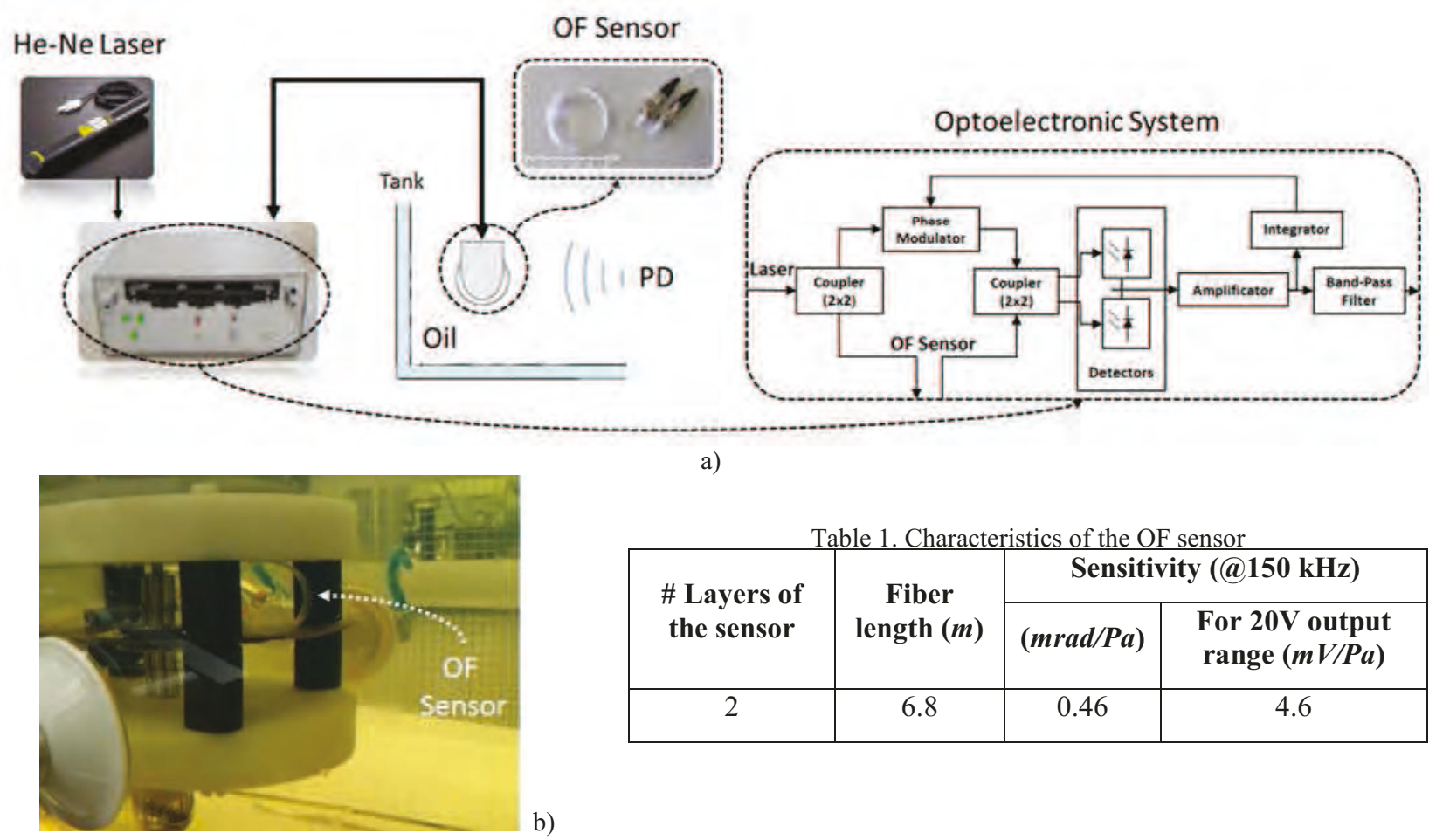

a)

Figure 2. a) Instrumentation system based on OF sensors. b) Location of the OF sensor.

The PD detection setup includes an acoustic part and an electrical part. The first one is constituted by a multichannel measurement system and a set of acoustic sensors. The multichannel system has conditioning and acquisition hardware and processing software (LabVIEW). In this case, four PZT ultrasonic sensors (R15i-AST) fixed to the outer face of the walls of the tank and a OF sensor inside have been used. The electrical part is formed by PD detector system (TechImp) and a laptop with detecting software (PD Base II-TechImp). 
Figure 3 shows the entire system for the HVDC generation and the detection of PD. The generation setup consists of a mixed power HV transformer, DC power, an AC regulator and the DUT. This kind of transformer allows working in AC, $\mathrm{DC}$ and $\mathrm{AC}+\mathrm{DC}$ conditions.

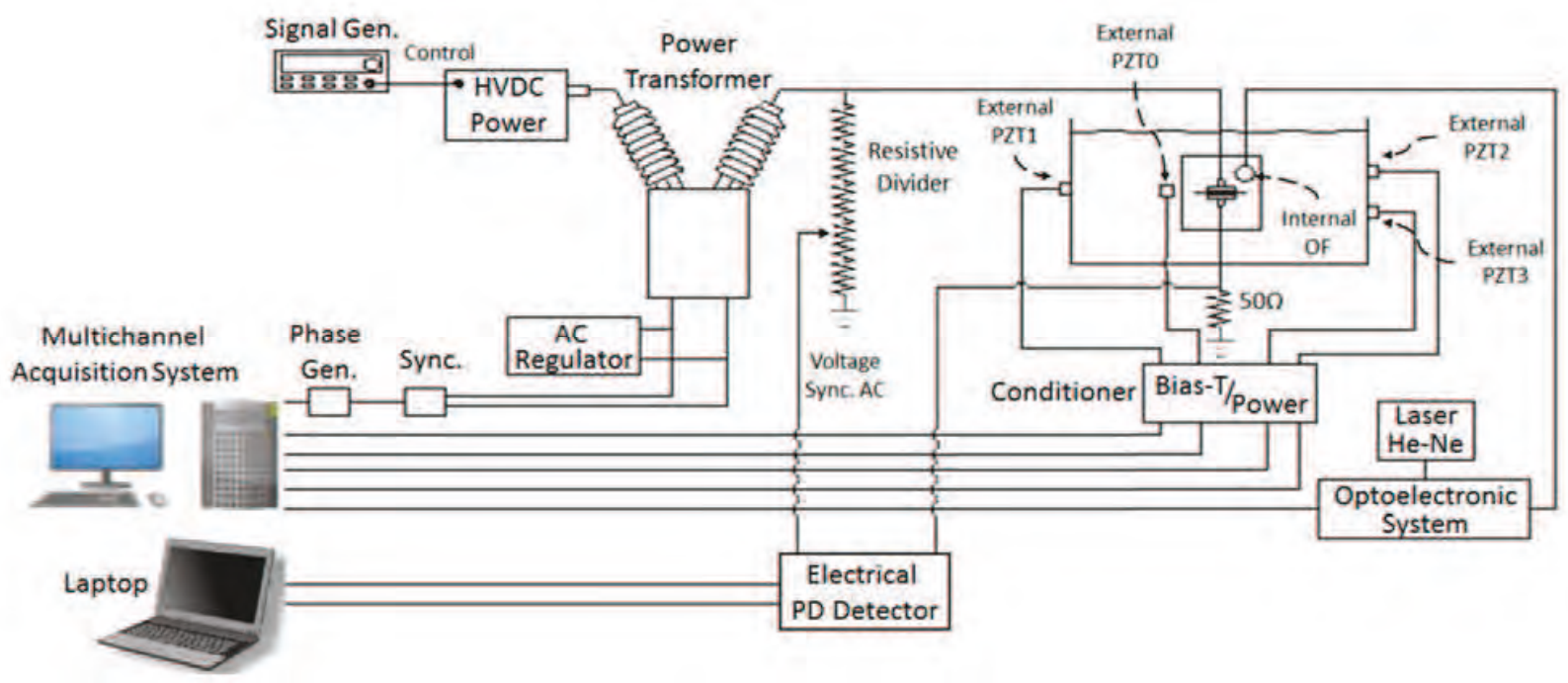

Figure 3. HVDC setup for the generation and detection of PD.

\section{PD MEASUREMENTS IN HVDC CONDITIONS}

In the experimental setup shown in Figure 3, PD measurements in HVDC conditions were done. However, since the inception voltage of PD is heavily dependent on the moisture of the oil and the impregnation level of the pressboard in oil, the pressboard immersed in the mineral oil was previously submitted to an extraction process of air and water by means of vacuum. The better insulation conditions as possible are achieved by these means. It is also important to highlight that the presence of the OF sensor in the DUT has not modified the inception voltage of PD.

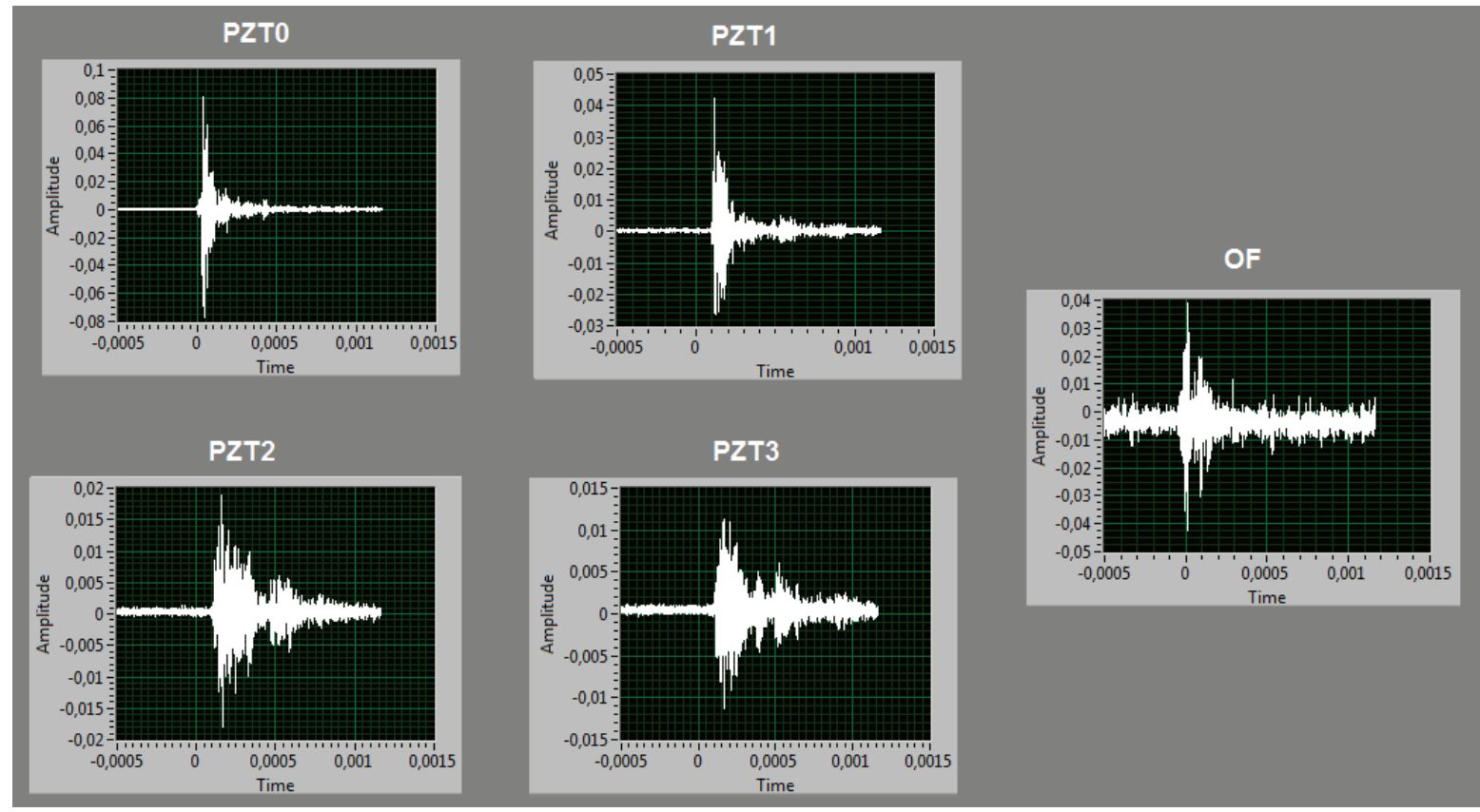

Figure 4. Results of PD measurements in HVDC conditions. [Time in s, Amplitude in V] 
A comparative study of the different techniques of PD detection (electrical and acoustic) was realized, analyzing the recorded signals by each sensor as the DC voltage increased.

The OF sensor detects acoustic signals form PD over the threshold of $2 \mathrm{~Pa}(9 \mathrm{mV}$ pk). Figure 4 shows a detail of the results obtained in the DP measurements where the OF sensor detected $9 \mathrm{~Pa}(40 \mathrm{mV}$ pk). The response of the OF sensor was not as good as expected with regards to acoustic PZT sensors, however it was able to detect PD where the electrical method could not. PD were gotten around $25 \mathrm{kV}_{\mathrm{DC}}$ due to the good insulation level.

\section{CONCLUSIONS}

A complete system of PD detection in HVDC transformers was developed in this work, which integrates in the same system different techniques of PD detection, electrical and acoustic, and it is able to monitor signals received by several types of sensors at the same time. It is worth to mention that an OF sensor for acoustic detection of PD was integrated in the HVDC experiments and compared to other detection methods.

The analysis of the recorded PD signals shows that the OF sensor provides information that helps to the assessment of the type and location of PD, not only in hard conditions of HV, but in the peculiar situation of HVDC. It contributes to another perspective that can be decisive in determining the reliability of the electrical insulation.

\section{ACKNOWLEDGEMENTS}

This work was supported by the Spanish Ministry of Science and Innovation under the Project DPI2009-14628-C03-01 and the scholarship FPI BES-2010-042083 and the Spanish Ministry of Economy and Competitiveness under the visiting researcher grant EEBB-I-2013-07675. C.G. Azcárraga-Ramos gratefully acknowledges the Instituto de Investigaciones Eléctricas for a study leave and the Mexican Science Council (CONACYT) for financially supporting his graduate studies. PD tests have been made in the High Voltage and Material Engineering Laboratory of the University of Bologna.

\section{REFERENCES}

[1] Morshuis, P. H. F. and Smit, J. J., "Partial Discharges at dc Voltage: Their Mechanism, Detection and Analysis," IEEE Transactions on Dielectrics and Electrical Insulation 12(2), 328-340 (2005).

[2] Piovan, U., "Insulation systems for HVDC transformers: present configurations, trends, challenges, solutions and open points," Proc. IEEE International Conference on Solid Dielectrics, 254-257 (2013).

[3] Lundgaard, L. E., "Partial Discharge XIII, Acoustic Partial Discharge Detection-Fundamental Considerations," IEEE Electrical Insulation Magazine 8(4), 25-31 (1992).

[4] Lundgaard, L. E., "Partial Discharge XIV, Acoustic Partial Discharge Detection-Practical Application," IEEE Electrical Insulation Magazine 8(5), 34-43 (1992).

[5] Eleftherion, P. M., "Partial Discharge XXI, Acoustic Emission-Based PD Source Location In Transformers," IEEE Electrical Insulation Magazine 11(6), 22-26 (1995).

[6] Rohwetter, P., Kielau, T., Lothongkam, C., Heidmann, G. and Habel,W., "All fibre-optic simultaneous detection of optical and acoustic emission from partial discharges in silicone elastomer," Proc. SPIE 8421-B9, 1-4 (2012).

[7] Tong, X., Ji, T., Wei, W., Wen, C., Zhu, X. and Leng, Z., “An optical fiber F-P acoustic emission sensor system for the detection of steel crack initiation," Proc. SPIE 8421-AG, 1-4 (2012).

[8] Fracarolli, J.P.V., Floridia, C., Dini, D.C., Rosolem, J.B. and Teixeira, R.M., "Fiber Optic Interferometric Method for Acoustic Emissions Detection on Power Transformer's Bushing," SBMO/IEEE MTT-S International Microwave \& Optoelectronics Conference, 1-5 (2013).

[9] Posada, J. E., Garcia-Souto, J. A. and Rubio-Serrano, J., "Multichannel optical-fibre heterodyne interferometer for ultrasound detection of partial discharges in power transformers," Measurement Science and Technology 24(9), 1-9 (2013).

[10] Posada-Roman, J., Garcia-Souto, J. A. and Rubio-Serrano, J., "Fiber optic sensor for acoustic detection of partial discharges in oil-paper insulated electrical systems," Sensors 12 (4), 4793-4802 (2012). 